\title{
Transcription factor TBX18 promotes adult rat bone mesenchymal stem cell differentiation to biological pacemaker cells
}

\author{
YANJUN LI, MEI YANG, GEGE ZHANG, LE LI, BINGJIE YE, CONGXIN HUANG and YANHONG TANG \\ Department of Cardiology, Renmin Hospital of Wuhan University, Cardiovascular Research Institute, \\ Wuhan University, Hubei Key Laboratory of Cardiology, Wuhan, Hubei 430060, P.R. China
}

Received August 8, 2016; Accepted November 1, 2017

DOI: $10.3892 /$ ijmm.2017.3259

\begin{abstract}
Bone mesenchymal stem cells (BMSCs) are currently considered the optimal stem cells for biological pacemaker cell transformation. The cardiac-specific transcription factor T-Box protein 18 (TBX18) is essential for sinoatrial node (SAN) formation, particularly formation of the head region that generates the electrical impulses that induce heart contraction. The present study aimed to confirm the effects of TBX18 on biological pacemaker differentiation of rat BMSCs. Flow cytometry was used to identify the surface markers of BMSCs, in order to acquire pure mesenchymal stem cells. Subsequently, BMSCs were transduced with TBX18 or green fluorescent protein adenovirus vectors. The effects of TBX18 were evaluated using SAN-specific makers including TBX18, $\alpha$-actin, cardiac troponin I, hyperpolarization-activated cyclic nucleotide-gated channel 4 and connexin 43 by reverse transcription-quantitative polymerase chain reaction, western blotting and immunofluorescence. The findings demonstrated that direct conversion of BMSCs to biological pacemaker cells via TBX18 is a feasible method in the field of cardiology.
\end{abstract}

\section{Introduction}

Gene- and cell-based hybrid treatment has received increased attention in the past decade with continuous refinement. In hybrid therapy, a human MSC loaded with the pacemaker gene hyperpolarization-activated cyclic nucleotide-gated channel 2 (HCN2) is implanted in the myocardium to induce the pacemaker function. The gene encoding transcription factor TBX18 has also been used to engineer phenotypic and functional pacemaker cells from myocardial cells in situ $(1,2)$. Stem cells possess the capacity to differentiate into numerous cellular phenotypes and are readily accessible; therefore, they are considered optimal candidates for cardiac pacing $(3,4)$. Bone

Correspondence to: Dr Yanhong Tang, Department of Cardiology, Renmin Hospital of Wuhan University, Cardiovascular Research Institute, Wuhan University, Hubei Key Laboratory of Cardiology, 238 Jiefang Road, Wuchang, Wuhan, Hubei 430060, P.R. China E-mail: wurmheart@vip.163.com

Key words: transcription factor T-Box protein 18, bone mesenchymal stem cells, differentiation, biological pacemaker, reprogramming mesenchymal stem cells (BMSCs) are a type of adult stem cell that have been widely used as cytoreagents for gene therapy (5).

BMSCs are heterogeneous cells derived from bone marrow cavities, which have various advantages compared with other types of stem cell. Notably, BMSCs are able to differentiate into various cell types in vitro. Based on these characteristics, mesenchymal stem cells can be used to deliver biological pacemaker genes, in order to induce the formation of functional and phenotypic pacemaker cells (6-9).

During cardiogenesis, human T-box transcription factors, including T-Box protein 3 (TBX3), TBX5 and TBX18 serve a prominent role in development of the sinoatrial node (SAN), particularly TBX18, which is a gene necessary for early SAN specification. Mesenchymal progenitor cells that express the TBX18 transcription factor are associated with generation of the SAN, particularly formation of the head region, which not only accounts for $\sim 75 \%$ of SAN volume, but also produces pacemaker activity in the early phase of embryonic heart formation (10-12). TBX18 gene knock-out mice exhibit a marked reduction in the SAN head region (13). In addition, a proof-of-concept study successfully converted postnatal rat ventricular myocytes to induced pacemaker cells via TBX18 expression (14). These findings may provide information regarding a novel method for generation of a biological pacemaker.

To the best of our knowledge, direct evidence that indicates the transformation of BMSCs to biological pacemaker cells via the TBX18 transcription factor is currently lacking. In the present study, TBX18 was expressed via adenovirus vectors to identify whether a single transcription factor is sufficient to induce differentiation of phenotypic and functional pacemakers. The present study aimed to provide an increased array of treatment options for gene and cell therapies.

\section{Materials and methods}

Animals. Adult male Sprague-Dawley rats $(\mathrm{n}=30$; age, 2 months; weight, 160-200 g) were purchased from the Center for Disease Control and Prevention of Hubei Province (Hubei, China). The rats were housed in micro-isolators under specifc pathogen-free conditions, in a climate-controlled enrivonment with an ambient temperature of $24^{\circ} \mathrm{C}$ and a $12: 12 \mathrm{~h}$ light/ dark cycle. They were fed with standard laboratory chow and given sterilized water. The present study was approved by the Experimental Animal Committee of Wuhan University 
Table I. Primer sequences used in the study.

\begin{tabular}{llrr}
\hline Gene & \multicolumn{1}{c}{ Primer sequences (5'-3') } & Accession no. & Size (bp) \\
\hline R- $\alpha$-actin & $\begin{array}{l}\text { Forward: GAGCACGGCATTATCACCAAC } \\
\text { Reverse: CAGAACAATGCCTGTGGTTCTC }\end{array}$ & NM_019183.1 & 246 \\
R-HCN4 & $\begin{array}{l}\text { Forward: CACTAAGGGCAACAAGGAGACC } \\
\text { Reverse: GGTAGTTGAAGACGCCTGAGTTG }\end{array}$ & NM_021658.1 & 281 \\
R-CX43 & $\begin{array}{l}\text { Forward: GCTGGTGGTGTCCTTGGTGT } \\
\text { Reverse: GGAGGAGACATAGGCGAGAGTG }\end{array}$ & NM_012567.2 & 213 \\
R-GAPDH & $\begin{array}{l}\text { Forward: CGCTAACATCAAATGGGGTG } \\
\text { Reverse: TTGCTGACAATCTTGAGGGAG }\end{array}$ & NM_017008.3 & 201 \\
H-TBX18 & $\begin{array}{l}\text { Forward: ACGTCATCCGTAAAGACTGTGG } \\
\text { Reverse: AGTCCGTAGTGATGGTCGCC }\end{array}$ & NM_001080508.2 & 251 \\
H-GAPDH & $\begin{array}{l}\text { Forward: GGTCGGAGTCAACGGATTTG } \\
\text { Reverse: GGAAGATGGTGATGGGATTTC }\end{array}$ & NM_002046.3 & 218 \\
\hline
\end{tabular}

CX43, connexin 43; HCN4, hyperpolarization-activated cyclic nucleotide-gated channel 4; TBX18, T-Box protein 18.

(Hubei, China). All animals received care in accordance with the guidelines for animal care published by the United States National Institutes of Health (NIH) (Guide for the Care and Use of Laboratory Animals, Department of Health and Human Services, NIH Publication no. 86-23, revised 1985).

Isolation and purification of BMSCs. BMSCs were isolated and cultured as described by Huang et al (15) with some modifications. In addition, all attempts were made to minimize rat suffering. Briefly, following sacrifice, the femurs and tibias of rats were quickly stripped, and muscle and extraossial tissue were trimmed. A $5 \mathrm{ml}$ syringe equipped with complete culture medium [Dulbecco's modified Eagle's medium/nutrient mixture F-12 supplemented with $10 \%$ fetal bovine serum (both Gibco; Thermo Fisher Scientific, Inc., Waltham, MA, USA), $100 \mathrm{U} / \mathrm{ml}$ penicillin and $100 \mathrm{U} / \mathrm{ml}$ streptomycin] was inserted into the bone marrow cavity, so as to flush target bone marrow cells into culture dishes, which were cultured in an atmosphere containing $5 \% \mathrm{CO}_{2}$ at $37^{\circ} \mathrm{C}$. The medium was initially substituted at $48 \mathrm{~h}$ and was then changed every 3 days. Once the cells reached $80 \%$ confluence, adherent cells were trypsinized with $0.25 \%$ trypsin solution and passaged. Cells from passages 3-5 were available for use in the following experiments.

Characterization of BMSCs by flow cytometry. BMSCs within passages 3-5 were harvested by trypsinization, and the detached cells were resuspended in PBS.Subsequently, approximately $1 \times 10^{6}$ cells were stained with the following antibodies: Alexa Fluor ${ }^{\circledR} 647$ Hamster Anti-Rat cluster of differentiation (CD)29 (562153, 1:100), phycoerythrin (PE)-Cy ${ }^{\mathrm{TM}} 7$ Mouse Anti-Rat CD90 (561404, 1:100) and fluorescein isothiocyanate (FITC) Mouse Anti-Rat CD45 (561867, 1:100) (all BD Biosciences, San Jose, CA, USA). Control samples were stained with Alexa Fluor ${ }^{\circledR}$ 647-conjugated hamster immunoglobulin ( $\mathrm{Ig}) \mathrm{M}$ isotype antibody $(562110,1: 100)$ or PE-Cy ${ }^{\mathrm{TM}} 7$-conjugated mouse IgG1 isotype antibody $(557872,1: 100)$ and FITC-conjugated mouse IgG1 isotype antibody (550616, 1:100) (all from BD Biosciences).
Whole incubations were performed at $4^{\circ} \mathrm{C}$ for $20 \mathrm{~min}$. After incubation, the cells were analyzed using a FACSCalibur flow cytometer (BD Biosciences).

Construction and purification of human TBX18 gene adenovirus vector. pHBAd-MCMV-GFP (HanBio Biotechnology Co., Ltd., Shanghai, China) was digested with BamHI and NotI. The ORF sequence of the human TBX18 gene (GenScript, Nanjing, China) was amplified by polymerase chain reaction (PCR). After enzyme digestion, gel extraction was performed. The digested fragment and vector were ligated to form pHBAd-MCMV-GFP-TBX18, which was then transformed into competent DH5 $\alpha$ cells (Tiangen, Beijing, China). Positive clones were identifed by liquid sequencing. Bacteria in liquid in the logarithmic growth phase were incubated at $37^{\circ} \mathrm{C}$ in $\mathrm{LB}$ culture medium with shaking at $300 \mathrm{x}$ g overnight. Large scale preperation of recombinant plasmid was conducted using the Plasmid Midi Preparation kit (Beijing CW Biotech Co., Ltd., Beijing, China). 293 cells (from our laboratory) were transfected with pHBAd-MCMV-GFP-TBX18 and the backbone vector pHBAd-BHG using Lipoflter ${ }^{\mathrm{TM}}$ (both from HanBio Biotechnology Co., Ltd.). The supernatant was harvested after virus amplifcation. Ad-GFP and Ad-TBX18 were measured as $110^{10} \mathrm{PFU} / \mathrm{ml}$ and were preserved at $-80^{\circ} \mathrm{C}$.

Transduction of BMSCs with hTBX18-expressing adenovirus vector. About (5-8) $\times 10^{5} \mathrm{BMSCs}$ were infected with pHBAd-MCMV-GFP-TBX18 or the pHBAd-MCMV-GFP empty vector at a multiplicity of infection (MOI) of 20, 50, 80 and 100 for $2 \mathrm{~h}$ at $37^{\circ} \mathrm{C}$, after which the medium was replaced with complete culture medium. Transduction efficiency was estimated according to the proportion of GFP-positive cells. After 24 and 48 h, inverted fluorescence microscopy (IX51; Olympus Corporation, Tokyo, Japan) was used to detect GFP expression. A total of 2 days postinfection, cells were accumulated for evaluation of hTBX18 expression by western blotting and reverse transcription-quantitative PCR (RT-qPCR). 

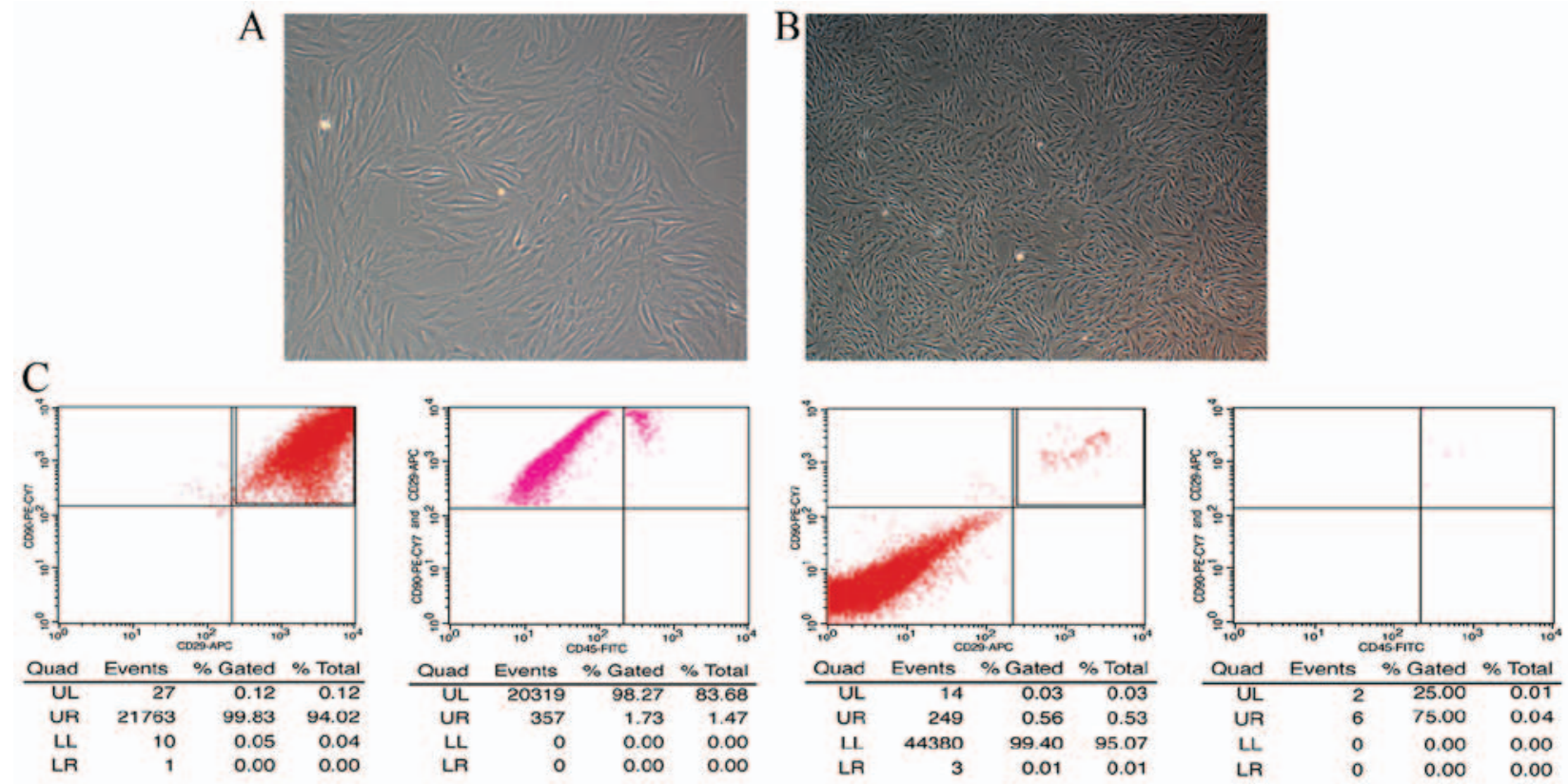

Figure 1. Characterization of BMSCs. (A) BMSCs at passage 3 exhibited unified spindle cell morphology, and tightly adhered to the bottom of the culture dish (magnification, x100). (B) Cultured BMSCs, 80-90\% confluent (magnification, x40), were applied for subsequent experiments. (C) Cell surface markers of BMSCs . BMSCs, bone mesenchymal stem cells; CD, cluster of differentiation.

Total RNA isolation and RT-qPCR. Total cellular RNA was extracted from BMSCs using TRIzol reagent (Invitrogen; Thermo Fisher Scientific, Inc.) according to the manufacturer's protocol. Subsequently, RT was conducted using the PrimeScript ${ }^{\mathrm{TM}}$ RT reagent kit (Takara Biotechnology, Ltd., Dalian, China) in a $20 \mu \mathrm{l}$ mixture. RT-qPCR was performed using SYBR ${ }^{\circledR}$ Premix Ex Taq ${ }^{\mathrm{TM}}$ kit (Takara Biotechnology,Ltd.) as follows: $95^{\circ} \mathrm{C}$ for $1 \mathrm{~min}$ followed by 40 cycles of $95^{\circ} \mathrm{C}$ for $15 \mathrm{sec}, 58^{\circ} \mathrm{C}$ for $20 \mathrm{sec}$ and $72^{\circ} \mathrm{C}$ for $45 \mathrm{sec}$ and a final extension at $60^{\circ} \mathrm{C}$ for $10 \mathrm{~min}$. GAPDH was used as a reference gene. The results of RT-qPCR were analyzed using the $2^{-\Delta \Delta C q}$ method (16). To ensure accuracy, each sample was analyzed $\geq 3$ times. The primers used in this experiment are presented in Table I.

Western blot analysis. The cells were harvested using RIPA lysis buffer (Beyotime Institute of Biotechnology, Haimen, China). Equal amounts of protein $(40 \mu \mathrm{g})$ were loaded onto a gel for $15 \%$ sodium dodecyl sulphate-polyacrylamide gel electrophoresis (SDS-PAGE), and the seperated proteins were transferred to a nitrocellulose membrane, and were subsequently incubated with primary antibodies overnight at $4^{\circ} \mathrm{C}$. Goat-anti-TBX18 polyclonal primary antibody (sc-17869, 1:200; Santa Cruz Biotechnology, Inc., Dallas, TX, USA) was applied to detect TBX18 protein expression $48 \mathrm{~h}$ post-transduction. Rabbit anti- $\alpha$-actin monoclonal antibody (ab156302, $1: 3,000)$, mouse anti-cardiac troponin I (cTnI) monoclonal antibody (ab19615, 1:1,000), rat anti-hyperpolarization-activated cyclic nucleotide-gated channel 4 (HCN4) monoclonal antibody (ab32675, 1:500) and rabbit anti-connexin 43 (CX43) polyclonal antibody (ab11370, 1:2,000) (all Abcam, Cambridge, MA, USA) were applied to detect biological pacemaker induction of BMSCs after 1 week of culture. After washing, the membranes were incubated with 1:10,000 horseradish peroxidase (HRP)-rabbit anti-goat (14-13-06), HRP-goat anti-rabbit (074-1506), anti-rat (14-16-06) or anti-mouse IgG (074-1806) (all KPL, Inc., Gaithersburg, MD, USA) secondary antibodies for $30 \mathrm{~min}$ at room temperature. Enhanced chemiluminescence detection (ECL; Beyotime Institute of Biotechnology) was then performed. GAPDH was used as a loading control. All western blot analyses were repeated $>3$ times.

Immunofluorescence staining. A total of 1 week post-transduction, immunofluorescence staining was performed on cultured cells. Briefly, approximately (5-8) $\times 10^{5}$ cells grown on glass coverslips were fixed with $4 \%$ paraformaldehyde for $20 \mathrm{~min}$ at room temperature. Following permeabilization with $0.1 \%$ Triton X-100, cells were incubated overnight with rat anti-HCN4 monoclonal primary antibody (ab32675, 1:50; Abcam) at $4^{\circ} \mathrm{C}$ and were then incubated with Cy3-labeled goat anti-rat secondary antibody (AS-1111, 1:50; Aspen Biotechnology, Hubei, China) for $50 \mathrm{~min}$ at room temperature. Nuclei were also stained with 4',6-diamidino-2-phenylindole for $5 \mathrm{~min}$. Fluorescent images were acquired using an inverted fluorescent microscopy.

Statistical analysis. The reported data are expressed as the means \pm SD. The statistical signifcance of the differences between two groups was determined using the Student's t-test. Comparisons among 3 groups were made using one-way analysis of variance (ANOVA). A P-value $<0.05$ was considered to indicate a statistically significant difference.

\section{Results}

Morphological characteristics andidentification of BMSCs. When initially seeded, BMSCs appeared round in shape. After $48 \mathrm{~h}$, cells became adherent, and exhibited triangular, fusiform or spindle shapes. These colonies fused after 6-8 days of culture; the majority of cultured cells exhibited uniform spindle-like morphology. As the number of passages increased, sub-cultured cells were more purified, homogenous and spindle-like (Fig. 1A and B). 
A

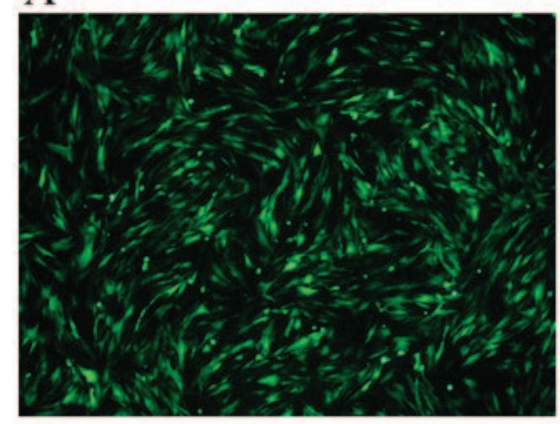

$\mathrm{C}$

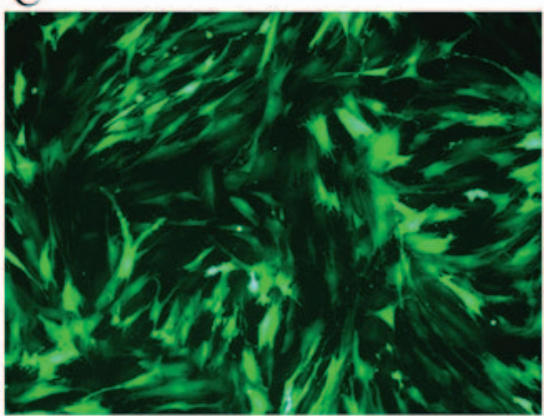

B

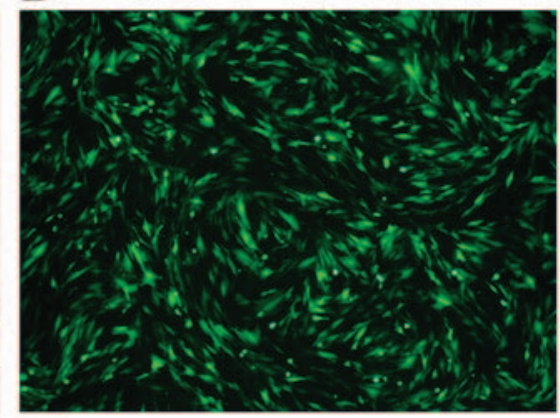

D

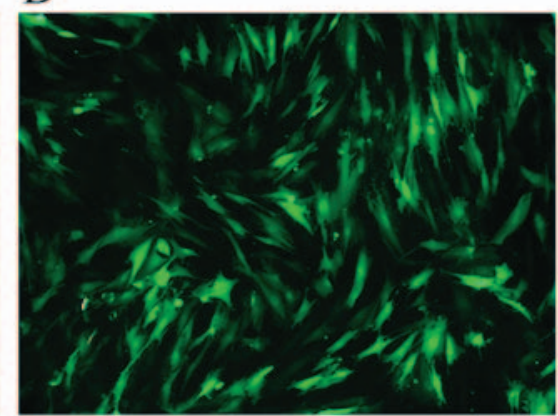

Figure 2. Green fluorescence of BMSCs (passage 3) transduced with TBX18 or GFP for 24 and 48 h under an Olympus IX51 inverted fluorescence microscope. (A) TBX18-transduced BMSCs at $24 \mathrm{~h}$ (magnification, x40). (B) GFP-transduced BMSCs at $24 \mathrm{~h}$ (magnification, x40). (C) TBX18-transduced BMSCs at $48 \mathrm{~h}$ (magnification, x100). (D) GFP-transduced BMSCs at $48 \mathrm{~h}$ (magnification, x100). BMSCs, bone mesenchymal stem cells; GFP, green fluorescent protein; TBX18, T-box protein 18 .

Flow cytometric analysis. To identify BMSC phenotypes, flow cytometry was conducted. The results indicated that BMSCs expressed stromal cell markers CD29 and CD90 ( $\leq 99 \%)$; however, almost no expression of the hematopoietic marker, CD45, was detected (Fig. 1C). These findings suggested that the cells tested were purified BMSCs.

Transduction of BMSCs with pHBAd-MCMV-GFP-TBX18 or pHBAd-MCMV-GFP. Through continuous attempts, the present study confirmed that the appropriate MOI was 100 , which was the lowest adenovirus titer that generated optimal infection efficiency. Post-transduction for $24 \mathrm{~h}$, green fluorescence could be detected under fluorescent microscopy. Under this condition, the transduction efficiency was 97-99\% (Fig. 2A and B). The expression levels and high infection efficiency peaked at $48 \mathrm{~h}$ and then faded gradually (Fig. 2C and D). These findings confirmed that the plasmids had been successfully transduced into the BMSCs.

Identification of target protein expression by western blotting and $R T-q P C R$. A total of $48 \mathrm{~h}$ after lipofection, transgene expression was examined by RT-qPCR and western blotting. The protein expression levels of TBX18 in BMSCs are presented in Fig. 3A; the results indicated that the transduced cells successfully expressed TBX18 protein. The protein expression levels of TBX18 were normalized to GAPDH (Fig. 3B). The results of RT-qPCR detection of TBX18 mRNA, relative to GAPDH, are presented in Fig. 3C. These results indicated that pHBAd-MCMV-GFP-TBX18 was successfully introduced into BMSCs.

RT-qPCR analysis of genes associated with cardiac pacing in BMSCs. The expression levels of target genes associated with biological pacemaker development were detected in BMSCs infected with pHBAd-MCMV-GFP-TBX18. The genes detected in the present study included $\alpha$-actin, HCN4 and CX43. As shown in Fig. 3D, the mRNA expression levels of $\alpha$-actin and HCN4 were significantly increased in BMSCs infected with TBX18 adenovirus (T group) compared with in the empty plasmid group (G group) and the blank group (B group) $(\mathrm{P}<0.05)$. Conversely, the expression levels of CX43 were significantly lower in the $\mathrm{T}$ group compared with in the $\mathrm{G}$ and $\mathrm{B}$ groups $(\mathrm{P}<0.05)$. No significant differences were detected between the $\mathrm{G}$ and $\mathrm{B}$ groups.

Western blot analysis of target protein expression. A total of 1 week post-transduction, total proteins were extracted from cells in the T, G and B groups. Western blotting was used to detect the protein expression levels of $\alpha$-actin, cTnI, HCN4 and CX43. The results indicated that $\alpha$-actin, cTnI and HCN4 expression levels in the $\mathrm{T}$ group were significantly upregulated compared with in the $\mathrm{G}$ and $\mathrm{B}$ groups $(\mathrm{P}<0.05)$. Conversely, CX43 expression was significantly downregulated in the $\mathrm{T}$ group compared with in the $\mathrm{G}$ and $\mathrm{B}$ groups $(\mathrm{P}<0.05$; Fig. $3 \mathrm{E})$. No significant differences were detected between the $G$ and $B$ groups.

Immunocytochemical analysis of BMSCs following transgene induction. After being cultured for 1 week, some differentiated cells developed the biological pacemaker phenotype. As demonstrated in Fig. 4A, TBX18-transduced BMSCs co-expressed HCN4 (red) and GFP (green) in vitro, indicating that the TBX18 adenovirus vector induced biological pacemaker differentiation of BMSCs. However, in the GFP-transduced group HCN4 protein expression (red) was barely detectable (Fig. 4B). 
A

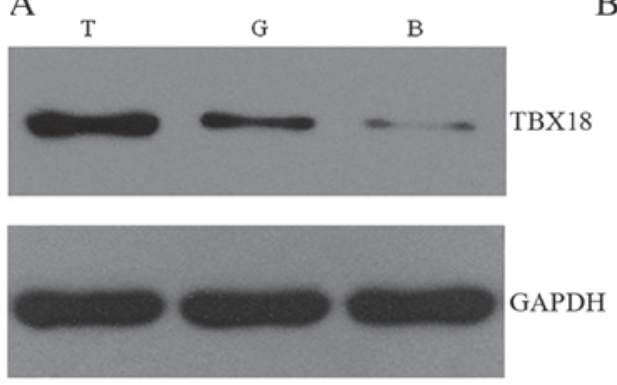

$\mathrm{D}$

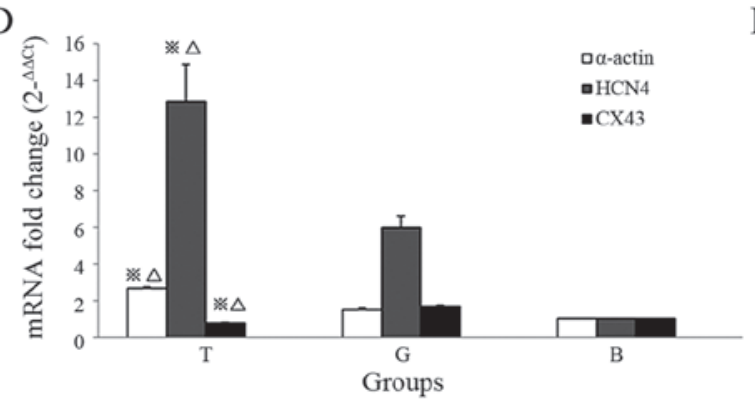

$\mathrm{B}$
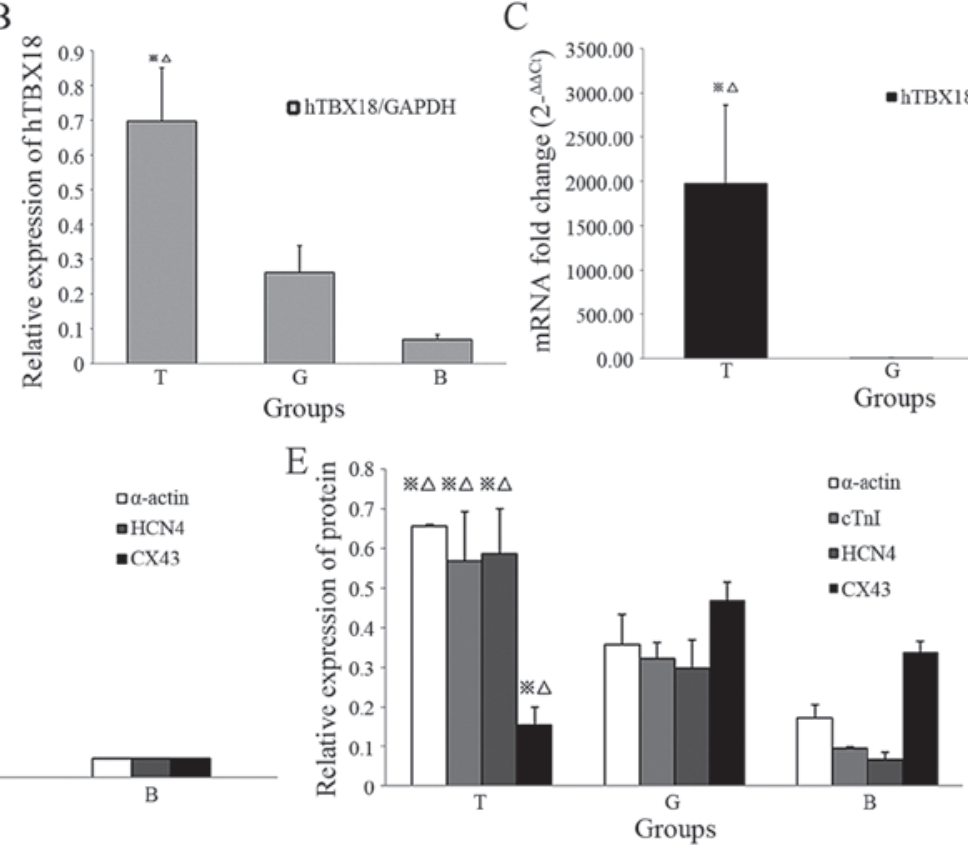

Figure 3. Western blotting and reverse transcription-quantitative polymerase chain reaction analysis of target protein and mRNA expression post-transduction. (A) pHBAd-MCMV-GFP-TBX18-transduced BMSCs displayed exogenous TBX18 expression, as determined by western blotting. (B) Protein expression levels of TBX18 were verified in differentiated cells by western blotting. (C) Alterations in the relative mRNA expression levels of TBX18 were compared between the T group and the G and B groups. (D) Quantitative analysis of the mRNA expression levels of $\alpha$-actin, HCN4 and CX43. (E) Western blotting detected increased $\alpha$-actin, $c T n I$ and $\mathrm{HCN} 4$ protein expression, and reduced $\mathrm{CX} 43$ protein expression, in the $\mathrm{T}$ group compared with in the $\mathrm{G}$ and $\mathrm{B}$ groups. ${ }^{\mathrm{P}}<0.05$, T group vs. G group; ${ }^{\circ} \mathrm{P}<0.05$, T group vs. B group. BMSCs, bone mesenchymal stem cells; B, blank group; cTnI, cadiac troponin I; CX43, connexin 43; G, GFP group/empty plasmid group; GFP, green fluorescent protein; HCN4, hyperpolarization-activated cyclic nucleotide-gated channel 4; hTBX18, human TBX18; T, TBX18 group; TBX18, T-box protein 18 .

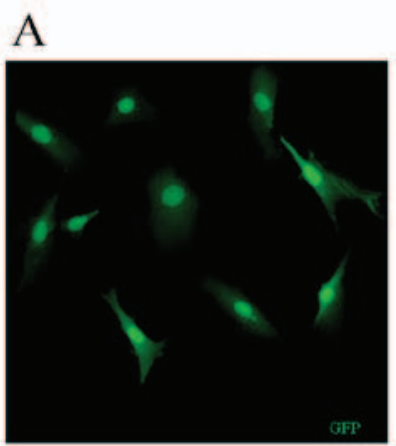

B

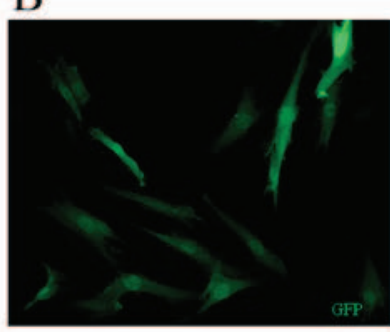

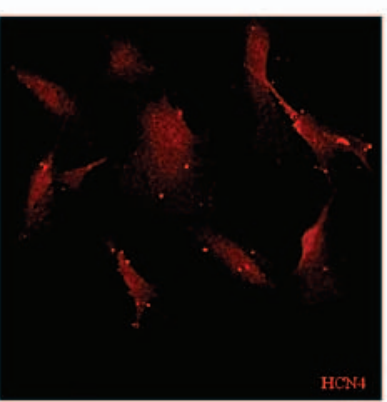
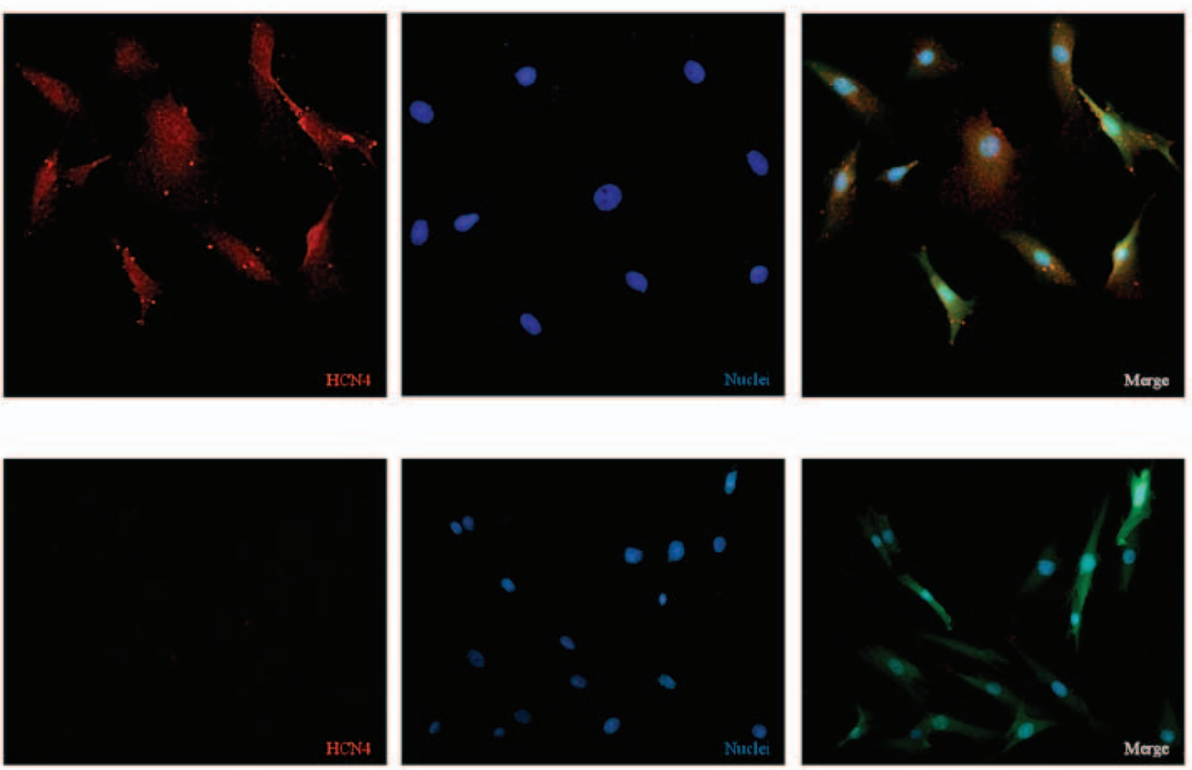

Figure 4. Fluorescence images of BMSCs post-transduction (magnification, x200). (A) BMSCs were transduced with pHBAd-MCMV-GFP-TBX18, and HCN4 and GFP expression were detected. Nuclei were stained blue with DAPI. (B) BMSCs transduced with the pHBAd-MCMV-GFP control vector expressed GFP but not HCN4. BMSCs, bone mesenchymal stem cells; DAPI, 4',6-diamidino-2-phenylindole; GFP, green fluorescent protein; HCN4, hyperpolarization-activated cyclic nucleotide-gated channel 4; TBX18, T-box protein 18.

\section{Discussion}

As a consequence of the advanced progress in the field of molecular biology, gene reprogramming techniques have been used in various aspects of biology. BMSCs exhibit numerous differentiative abilities in vivo and in vitro $(17,18)$. Notably, mesenchymal stem cells can inhibit T-cell proliferation, so as to restrain immunoreactivity of the host (19). BMSCs loaded 
with the biological pacemaker genes HCN4 or HCN2 have been successfully implanted into the myocardium of large animals to induce pacemaker function (20-23).

BMSCs are easy to modify at the genetic level. The present study introduced the TBX18 transcription factor into BMSCs to determine whether this gene is able to accomplish the direct conversion of BMSCs to biological pacemaker cells. TBX18 can not only induce the direct conversion of neonatal rat ventricular myocytes to pacemaker cells, but also produces stable pacemaker activity for 2 weeks via injection of TBX18 adenovirus in pigs, along with complete heart block (24). These findings suggested that TBX18 may be an optimal candidate gene in the biological pacemaker process. In the present study, on the 7 th day of culture, positive results were detected, and the outcomes were evaluated with western blotting, RT-qPCR and immunofluorescence.

To identify whether TBX18 may induce the myocardial phenotype of BMSCs, the cells were incubated with antibodies against cardiomyocyte-associated proteins, including cTnI and $\alpha$-actin, which are myocardium-specific markers. cTnI is one of three subunits of troponin (cTnI, cTnT and cTnC), which is located in the thin filaments (25). cTnI inhibits the connection between myosin and actin via intracellular $\mathrm{Ca}^{2+}$ to regulate muscle contraction and relaxation; mutations in cTnI result in hypertrophic cardiomyopathy (26). $\alpha$-actin is known as cardiac structural gene, which is expressed in mouse cardiac tissue during embryogenesis and can provide redundant function (27). In the present study, western blotting and RT-qPCR detected cTnI and $\alpha$-actin expression in TBX18-transduced BMSCs, which is consistent with the features of the SAN.

Notably,HCN4 expression was detected in the induced cells in the present study. The SAN is located in the right atrium adjacent to the superior vena cava, and is responsible for heartbeat initiation (28). HCN4 is an important HCN isoform, which accounts for $>80 \%$ of the total amount of HCN mRNA $(29,30)$. Furthermore, HCN4 expression is the most typical characteristic of pacemaker cells. Furthermore, HCN4 expression is the most typical characteristic of pacemaker cells. In the present study, HCN4 expression was detected through western blotting, RT-qPCR and immunofluorescence. The present study demonstrated that the expression levels of cTnI, $\alpha$-actin and HCN4 were significantly increased in TBX18-transduced cells. However, CX43 expression levels were markedly downregulated in the TBX18-transduced groups compared with in the GFP-transduced and blank groups. These findings were consistent with those of Kapoor et al (31). TBX18 may repress the CX43 promoter directly, further to the transcript and protein levels, other connexins like CX30.2, CX40 and CX45 promotes the slow action potential propagation. The characteristics of the TBX18-transduced BMSCs were similar to the functional features of the SAN, which include slow contractibility and slow-propagating connexins compared with the surrounding myocardium.

For high-efficiency transduction, adenovirus vectors were selected as ideal gene therapy vehicles in the present study. In previous studies, adenovirus vectors have been used for a large amount of transgene therapies, due to their ease of production, high titers, stable inheritance, sufficient transduction efficiency and early peak expression compared with other vectors (32-35). However, adenovirus-mediated gene expression may not last a long period of time; peaking at $\sim 1$ week, declining at
3-5 weeks and vanishing at 10 weeks (36). Consequently, adenovirus vectors are appropriate for temporary applications that have no demand for long-term gene expression. In addition, adenovirus vectors are likely to induce inflammatory and immune responses; therefore, their permanent safety and efficacy remains to be improved.

In conclusion, the present study indicated that expression of the transcription factor TBX18 is capable of initiating differentiation of BMSCs into biological pacemaker cells. This discovery represents a possible therapeutic alternative to electronic devices. However, long-term practice in large animals is required to evaluate the safety prior to application in patients with sinus dysfunction.

\section{Acknowledgements}

Flow cytometry conducted in the present study was supported by Central Laboratory, Renmin Hospital, Wuhan University (Wuhan, China). The present study was supported by the Fundamental Research Funds for the Central Universities of China (grant nos. 2042015kf0229 and 2042014 kf0306).

\section{References}

1. Rosen MR: Gene therapy and biological pacing. N Engl J Med 371: 1158-1159, 2014.

2. Chauveau S, Brink PR and Cohen IS: Stem cell-based biological pacemakers from proof of principle to therapy: A review. Cytotherapy 16: 873-880, 2014.

3. Cai B, Li J, Wang J, Luo X, Ai J, Liu Y, Wang N, Liang H, Zhang M, Chen N, et al: microRNA-124 regulates cardiomyocyte differentiation of bone marrow-derived mesenchymal stem cells via targeting STAT3 signaling. Stem Cells 30: 1746-1755, 2012.

4. Psaltis PJ, Zannettino AC, Worthley SG and Gronthos S: Concise review: mesenchymal stromal cells: potential for cardiovascular repair. Stem Cells 26: 2201-2210, 2008.

5. Hamada H, Kobune M, Nakamura K, Kawano Y, Kato K, Honmou O, Houkin K, Matsunaga T and Niitsu Y: Mesenchymal stem cells (MSC) as therapeutic cytoreagents for gene therapy. Cancer Sci 96: 149-156, 2005.

6. Zhou YF, Yang XJ, Li HX, Han LH and Jiang WP: Mesenchymal stem cells transfected with HCN2 genes by LentiV can be modified to be cardiac pacemaker cells. Med Hypotheses 69: 1093-1097, 2007.

7. Yang XJ,Zhou YF, Li HX, Han LH and Jiang WP: Mesenchymal stem cells as a gene delivery system to create biological pacemaker cells in vitro. J Int Med Res 36: 1049-1055, 2008.

8. Ma J, Zhang C, Huang S, Wang G and Quan X: Use of rats mesenchymal stem cells modified with $\mathrm{mHCN} 2$ gene to create biologic pacemakers. J Huazhong Univ Sci Technolog Med Sci 30: 447-452, 2010.

9. Nong Y, Zhang C, Wei L, Zhang Z, Cheng J, Wen L and Song Z: In situ investigation of allografted mouse HCN4 gene-transfected rat bone marrow mesenchymal stromal cells with the use of patch-clamp recording of ventricular slices. Cytotherapy 15: 905-919, 2013.

10. Wiese C, Grieskamp T, Airik R, Mommersteeg MT, Gardiwal A, de Gier-de Vries C, Schuster-Gossler K, Moorman AF, Kispert A and Christoffels VM: Formation of the sinus node head and differentiation of sinus node myocardium are independently regulated by Tbx18 and Tbx3. Circ Res 104: 388-397, 2009.

11. McNally EM and Svensson EC: Setting the pace: Tbx3 and Tbx18 in cardiac conduction system development. Circ Res 104: 285-287, 2009.

12. Cho HC: Pacing the heart with genes: Recent progress in biological pacing. Curr Cardiol Rep 17: 65, 2015.

13. Greulich F, Trowe MO, Leffler A, Stoetzer C, Farin HF and Kispert A: Misexpression of Tbx18 in cardiac chambers of fetal mice interferes with chamber-specific developmental programs but does not induce a pacemaker-like gene signature. J Mol Cell Cardiol 97: 140-149, 2016. 
14. Kapoor N, Liang W, Marbán E and Cho HC: Direct conversion of quiescent cardiomyocytes to pacemaker cells by expression of Tbx18. Nat Biotechnol 31: 54-62, 2013.

15. Huang XP, Sun Z, Miyagi Y, McDonald Kinkaid H, Zhang L, Weisel RD and Li RK: Differentiation of allogeneic mesenchymal stem cells induces immunogenicity and limits their long-term benefits for myocardial repair. Circulation 122: 2419-2429, 2010.

16. Livak KJ and Schmittgen TD: Analysis of relative gene expression data using real-time quantitative PCR and the 2(-Delta Delta C(T)) Method. Methods 25: 402-408, 2001.

17. Karaoz E, Aksoy A, Ayhan S, Sariboyaci AE, Kaymaz F and Kasap M: Characterization of mesenchymal stem cells from rat bone marrow: Ultrastructural properties, differentiation potential and immunophenotypic markers. Histochem Cell Biol 132: 533-546, 2009

18. Ye NS, Chen J, Luo GA, Zhang RL, Zhao YF and Wang YM: Proteomic profiling of rat bone marrow mesenchymal stem cells induced by 5-azacytidine. Stem Cells Dev 15: 665-676, 2006.

19. Tokcaer-KeskinZ,Akar AR, Ayaloglu-Butun F, Terzioglu-KaraE, Durdu S, Ozyurda U, Ugur M and Akcali KC: Timing of induction of cardiomyocyte differentiation for in vitro cultured mesenchymal stem cells: A perspective for emergencies. Can J Physiol Pharmacol 87: 143-150, 2009.

20. Potapova I, Plotnikov A, Lu Z, Danilo P Jr, Valiunas V, Qu J, Doronin S, Zuckerman J, Shlapakova IN, Gao J, et al: Human mesenchymal stem cells as a gene delivery system to create cardiac pacemakers. Circ Res 94: 952-959, 2004

21. Plotnikov AN, Shlapakova I, Szabolcs MJ, Danilo P Jr, Lorell BH, Potapova IA, Lu Z, Rosen AB, Mathias RT, Brink PR, et al: Xenografted adult human mesenchymal stem cells provide a platform for sustained biological pacemaker function in canine heart. Circulation 116: 706-713, 2007.

22. Cheng J, Zhang Z, Wei L, Nong Y, Wen L, Qin Y and Song Z: Canine bone marrow mesenchymal stromal cells with lentiviral mHCN4 gene transfer create cardiac pacemakers. Cytotherapy 14: 529-539, 2012.

23. Wei L, Nong Y, Boli R, Cheng J, Zhang C, Zhou Y and Song Z: mHCN4 genetically modified canine mesenchymal stem cells provide biological pacemaking function in complete dogs with atrioventricular block. Pacing Clin Electrophysiol 36: 1138-1149, 2013.

24. Hu YF, Dawkins JF, Cho HC, Marbán E and Cingolani E: Biological pacemaker created by minimally invasive somatic reprogramming in pigs with complete heart block. Sci Transl Med 6: 245ra94, 2014.
25. Takahashi-Yanaga F, Morimoto S, Harada K, Minakami R, Shiraishi F, Ohta M, Lu QW, Sasaguri T and Ohtsuki I: Functional consequences of the mutations in human cardiac troponin I gene found in familial hypertrophic cardiomyopathy. J Mol Cell Cardiol 33: 2095-2107, 2001.

26. Ruan Z, Zhu L, Yin Y and Chen G: Overexpressing NKx2.5 increases the differentiation of human umbilical cord drived mesenchymal stem cells into cardiomyocyte-like cells. Biomed Pharmacother 78: 110-115, 2016.

27. Xu M, Wani M, Dai YS, Wang J, Yan M, Ayub A and Ashraf M: Differentiation of bone marrow stromal cells into the cardiac phenotype requires intercellular communication with myocytes. Circulation 110: 2658-2665, 2004.

28. Husse B and Franz WM: Generation of cardiac pacemaker cells by programming and differentiation. Biochim Biophys Acta 1948-1952: 2016, 1863.

29. Robinson RB and Siegelbaum SA: Hyperpolarization-activated cation currents: From molecules to physiological function. Annu Rev Physiol 65: 453-480, 2003.

30. Verkerk AO and Wilders R: Pacemaker activity of the human sinoatrial node: Effects of HCN4 mutations on the hyperpolarization-activated current. Europace 16: 384-395, 2014.

31. Kapoor N, Galang G, Marbán E and Cho HC: Transcriptional suppression of connexin 43 by TBX18 undermines cell-cell electrical coupling in postnatal cardiomyocytes. J Biol Chem 286: 14073-14079, 2011.

32. French BA, Mazur W, Geske RS and Bolli R: Direct in vivo gene transfer into porcine myocardium using replication-deficient adenoviral vectors. Circulation 90: 2414-2424, 1994.

33. Brenner M: Gene transfer by adenovectors. Blood 94: 3965-3967, 1999.

34. Wright MJ, Wightman LM, Lilley C, de Alwis M, Hart SL, Miller A, Coffin RS, Thrasher A, Latchman DS and Marber MS: In vivo myocardial gene transfer: Optimization, evaluation and direct comparison of gene transfer vectors. Basic Res Cardiol 96: 227-236, 2001

35. Li RA: Gene- and cell-based bio-artificial pacemaker: What basic and translational lessons have we learned? Gene Ther 19: $588-595,2012$.

This work is licensed under a Creative Commons Attribution-NonCommercial-NoDerivatives 4.0 International (CC BY-NC-ND 4.0) License. 